\title{
Museus Virtuais e Ecomuseus - Uma experiência fazendo uso de IoT
}

\author{
Nivaldo Henrique Nunes Bondança ${ }^{1}$, Rosana Teresinha Vaccare Braga ${ }^{2}$, \\ Kalinka Regina Lucas Jaquie Castelo Branco ${ }^{1}$
}

${ }^{1}$ Instituto de Ciências Matemáticas e de Computação - Universidade de São Paulo (ICMC/USP) Av. Trabalhador SãoCarlense, 400 - São Carlos - SP - Brazil

\{hick209@gmail.com, rtvb@icmc.usp.br, kalinka@icmc.usp.br\}

\begin{abstract}
A diversity of collections of inestimable historical and cultural value, containing objects of diverse natures, can be found scattered. In this sense, information and communication technologies tools, more specifically the Internet of Things (IoT), can allow improvement of educational activities and awareness of cultural and environmental heritage. The implementation of this work was done through the instantiation of the Museu da Fauna e Flora do ICMC-USP (MFF-ICMC), and was done using Progressive Web App (PWA) along with the Physical Web and Bluetooth low energy beacons. This paper seeks to explore a new way of interacting with the museum. It does that through an application that seeks to passively display to the user a link with a reference to more information about the element of the museum near to the user.
\end{abstract}

Resumo. Uma diversidade de acervos de inestimável valor histórico e cultural, contendo objetos de diversas naturezas, pode ser encontrado espalhado. Nesse sentido, ferramentas de Tecnologias de Informação e Comunicação (TICs), mais especificamente a Internet das Coisas (do Inglês Internet of Things - IoT), podem permitir melhoria de atividades de educação e sensibilização de patrimônios culturais e ambientais. A implementação deste trabalho se deu por meio da instanciação do Museu da Fauna e Flora do ICMC-USP (MFF-ICMC), e foi feita utilizando um Progressive Web App (PWA) em conjunto com a Web física e bluetooth low energy beacons. Neste artigo explora-se uma nova forma de interação com o museu, por meio de uma aplicação que busca mostrar, de forma passiva para o usuário, um link com uma referência para maiores informações sobre o elemento do museu próximo ao usuário.

\section{Introdução}

Com o uso de celulares conectados à internet junto de buscadores inteligentes, o acesso às informações, mesmo às pequenas curiosidades do dia-a-dia, está bastante facilitado. Não é mais necessário realizar grandes esforços para encontrar o que se busca, principalmente porque a Internet se tornou uma parte de nós, uma extensão de nossa capacidade [Sparrow et al. 2011].

Desse modo, o acesso ao conhecimento está mais fácil, entretanto, existem algumas barreiras para isso. Para acessar informações desejadas, é necessário ou saber o endereço da página que as contém, ou então conhecer o conjunto certo de palavras chaves para buscar o que precisa, supondo que o site esteja indexado propriamente pelos buscadores. Atritos como estes dificultam a difusão de informações. 
Nesse sentido, em algumas situações poderia ser interessante haver uma maneira da informação apenas ir até quem gostaria de acessá-las. Informações contextualizadas são interessantes, mas, assim como sugere o nome, são sensíveis ao contexto. Uma vez mudado o contexto, essa informação pode já não ser mais proveitosa.

Para uma pessoa que se encontra em um cinema, pode ser interessante ver mais informações sobre o filme em cartaz. No entanto, esta mesma informação, enquanto a pessoa se encontra no supermercado, já não tem o mesmo valor. Caso se considere que é interessante acessar informações mais contextualizadas, como isso deveria ser feito? Soluções devem ser exploradas.

O Museu da Fauna e Flora do ICMC da USP São Carlos (MFF-ICMC) possui um caso de uso interessante para acesso contextualizado à informação, sendo possível a utilização de uma aplicação que mostre os dados sobre o elemento do Museu do qual a pessoa se encontra próxima.

Esta é uma forma muito interessante de tornar um museu mais interativo, e no caso de museus virtuais, torná-los uma experiência mais real, pois o usuário poderá ver onde os elementos do museu se encontram no mundo real.

A Internet das Coisas (IoT - do inglês Internet of Things) [Atzori et al. 2010] oferece mecanismos para viabilizar essa ideia. Em se tratando do MFF-ICMC, torná-lo mais interativo por meio de dispositivos da IoT seria uma forna de melhor difundi-lo entre as pessoas que passam pelo campus, além de possibilitar a aquisição de novos apreciadores, especialmente se as informações forem apresentadas assim que a pessoa passa próxima de algum dos elementos do museu, como alguma árvore, que estão espalhados pelo campus.

O objetivo deste artigo é apresentar uma nova maneira de integrar dados do mundo virtual com objetos do mundo real, fornecendo informações mais contextualizadas ao usuário. Para isso, considerando que os usuários, em sua grande maioria, carregam consigo smartphones, desenvolveu-se um mecanismo usando beacons para facilitar o acesso ao acervo do MFF-ICMC.

Este artigo está dividido em 4 seções. Na seção 2 apresenta-se o MFF-ICC. Na Seção 3 é apresentada uma revisão da terminologia básica para o entendimento da proposta, abordando alguns dos principais conceitos relacionados às tecnologias utilizadas no desenvolvimento deste artigo. Na seção 4, descreve-se o desenvolvimento do aplicativo para o museu virtual, expondo os detalhes de algumas das decisões tomadas. Na Seção 5 comentam-se os resultados obtidos. Finalmente, na seção 6, são apresentadas as conclusões.

\section{Museu da Fauna e da Flora do ICMC}

Os desenvolvimentos no campo das TICs (Tecnologias da Informação e da Comunicação) trouxeram profundas mudanças nos últimos anos nas diversas áreas de conhecimento, com rebatimentos importantes no campo das políticas públicas, educação, comunicação, meio ambiente, entre outras. Observa-se um crescente interesse e acesso da sociedade em relação às TICs, especialmente no contexto das crianças e jovens em idade escolar.

Segundo a Pesquisa Nacional por Amostra de Domicílios - PNAD (IBGE, 2014), em 2013,48\% (31,2 milhões) do total de domicílios possuíam acesso à Internet, sendo que $42,4 \%$ (13,2 milhões) por meio de microcomputador e 3,6 milhões $(11,6 \%)$ por outros 
VI Congresso Brasileiro de Informática na Educação (CBIE 2017)

Anais dos Workshops do VI Congresso Brasileiro de Informática na Educação (WCBIE 2017)

meios (que incluem celulares). Os dados apontam que 32,4\% dos usuários da Internet com mais de 10 anos, o que equivale a 27,8 milhões de brasileiros, eram estudantes à época.

O CENSO Escolar da Educação Básica de 2012 (Instituto Nacional de Estudos e Pesquisas Educacionais Anísio Teixeira, 2013) demonstrou que a porcentagem de escolas com acesso à Internet em 2012 era de 45,8\% e 93,0\% nas escolas de ensino fundamental e médio da Rede Pública, respectivamente, e 90,5\% e 98,7\% respectivamente nas escolas de ensino fundamental e médio da Rede Privada.

No entanto, no contexto da educação básica, ainda é significativo o distanciamento entre a realidade escolar e o emprego das tecnologias disponíveis, devido principalmente às dificuldades dos sistemas de ensino em incorporá-las às atividades da escola. Além da Ciência da Informação, o campo teórico e metodológico da museologia muito tem a contribuir para a organização e disponibilização do conhecimento, principalmente, com a utilização da "nova museologia", movimento surgido na França, na década de 1970, alinhado com determinadas tendências museológicas, experienciadas desde os anos 1960. Uma dessas tendências é o "ecomuseu" que surge como uma possibilidade de democratização da memória, em uma perspectiva museológica inclusiva, com a participação crítica dos seus organizadores, gestores e usuários.

Conceitualmente, os ecomuseus se referem os parques naturais com preocupação ecológica, mas não se limita a isso a medida em que é pensado como um instrumento de mudança e, portanto, atende aos pressupostos da educação, em geral, e particularmente da educação ambiental, podendo contribuir para o desenvolvimento de meios para lidar com o patrimônio e a memória de determinada comunidade ou sociedade [Brulon 2015].

Pressupõe-se que o conceito de ecomuseu, por associar as noções de "comunidade" e de ambiente, apresenta maior possibilidade para se atribuir significado ao conhecimento, uma vez que este passa a ser organizado levando-se em consideração certos valores que são comuns gerando, assim, o sentimento de identidade e pertencimento. Essa identificação aumenta a possibilidade da sociedade contribuir na busca de solução para os problemas ambientais e sociais, principalmente se for facilitada por uma plataforma digital, que permite tornar o "museu" mais acessível e interativo.

A partir desse pressuposto, considera-se que um ecomuseu atua como apoio para a colaboração de membros da comunidade, tornando-se um patrimônio comunitário que passa a ser utilizado como suporte material e imaterial de ação e ajudam encontrar respostas sobre o passado, presente e futuro e, portanto, torna-se um importante instrumento educacional.

O projeto Museu da Flora e Fauna do $\mathrm{ICMC}^{1}$ (MFF-ICMC) é exemplo potencial do que ferramentas de TICs e ecomuseu podem criar na melhoria de atividades de educação e sensibilização de patrimônios culturais e ambientais, e portanto, muito alinhados ao contexto da biodiversidade, que é um dos principais patrimônios naturais.

O Museu da Fauna e Flora do ICMC é um projeto que registra espécies existentes no campus da USP em São Carlos, especialmente na região do ICMC, com intuito de promover a difusão da fauna e flora do Instituto, não só para a comunidade, mas para

\footnotetext{
${ }^{1}$ Museu da Fauna e Flora https://www.icmc.usp.br/cultura-e-extensao/ museu-da-fauna-e-flora
} 
VI Congresso Brasileiro de Informática na Educação (CBIE 2017)

Anais dos Workshops do VI Congresso Brasileiro de Informática na Educação (WCBIE 2017)

todas as pessoas interessadas, dentre as quais podem ser incluídos estudantes que fazem parte desde o ensino fundamental até mesmo os de pós-graduação.

\section{Terminologia básica}

\subsection{Tecnologia push}

A World Wide Web Consortium (W3C) ${ }^{2}$ é uma comunidade internacional que trabalha para organização da World Wide Web por meio do desenvolvimento de padrões para o desenvolvimento Web.

$\mathrm{Na}$ internet, é comum o servidor agir de forma passiva, apenas respondendo à requisições. A tecnologia push inverte este cenário. Nela o servidor é o agente que inicia a requisição, enviando informações para o cliente [Bell et al. 2011].

Um cenário comum onde pode-se encontrar esta tecnologia hoje em dia é nos celulares. Muitos recebem mensagens por meio da tecnologia push e usualmente as apresentam para o usuário em forma de notificação . Essas notificações são também denominadas notificação push.

\subsection{Service workers}

Service worker é uma tecnologia que permite que aplicações web usufruam do processamento em segundo plano do navegador de internet. O service worker provê ferramentas para uma aplicação web possa executar ações mesmo quando se encontra off-line.

Funcionalidades que normalmente são associadas aos aplicativos móveis nativos como: sincronizações periódicas em segundo plano, notificações push e capacidade de utilização do sistema quando off-line; hoje são possibilitadas na Web por meio de service workers. Pode-se dizer que service workers é uma tecnologia que busca fornecer uma experiência mais fluida para páginas da internet [Gaunt 2017].

A W3C possui um rascunho ${ }^{3}$ sobre a tecnologia de service worker, pois o padrão ainda está sendo definido e seus detalhes acertados. Apesar disto, a tecnologia já é suportada nos navegadores Chrome, Firefox e Opera o que, de acordo com o Canisue ${ }^{4}$, representa aproximadamente $73 \%$ do mercado dos browsers.

\subsection{Progressive web app}

Um Progressive web app (PWA) é uma aplicação web que faz uso do melhor das tecnologias que a web pode oferecer para obter comportamentos de aplicações nativas. A ideia de um PWA é ir adicionando progressivamente, funcionalidades que antes só eram possíveis em aplicativos nativos [Fransson and Driaguine 2017].

Algumas das principais características de um PWA são:

- Responsividade - Ser capaz de se adequar ao formato e tamanho de tela que estiver sendo utilizado.

\footnotetext{
${ }^{2}$ W3C https: / / www.w3.org/Consortium/

${ }^{3}$ W3C Service workers Draft https: //www.w3.org/TR/service-workers-1/

${ }^{4}$ Caniuse http: / / caniuse.com/ $\backslash \#$ feat=serviceworkers

${ }^{5}$ StatCounter GlobalStats http://gs. statcounter.com/
} 
VI Congresso Brasileiro de Informática na Educação (CBIE 2017)

Anais dos Workshops do VI Congresso Brasileiro de Informática na Educação (WCBIE 2017)

- Trabalhar off-line - Não necessita de uma conexão constante com a internet para funcionar.

- Parecer nativo - Se parece com um aplicativo nativo.

- Atualizado - Com auxílio do processo de atualização de cache do service worker e da própria web, o PWA deverá estar sempre na versão mais atualizada.

- Seguro - É considerado seguro, pois aplicações web executam, por padrão, em um ambiente isolado do resto do sistema.

- Descobrível - Ao utilizar o manifesto de aplicativo definido pelo W3C, os buscadores atuais já sabem identificar sua aplicação web como um "aplicativo", tornando-o mais fácil de ser descoberto através de uma busca.

- Instalável - Usuários devem poder "instalar" os aplicativos (PWAs) desejados.

- Linkável - Para compartilhar uma aplicação, basta enviar sua Uniform Resource Locator (URL) (seu link).

Pode-se entender que um PWA é um híbrido entre páginas da Web e aplicativos móveis, tentando combinar o melhor dos dois mundos.

\subsection{Bluetooth Low Energy}

Bluetooth Low Energy (BLE) ${ }^{6}$ é a especificação do Bluetooth desenvolvida pensando no mercado de IoT [Dasgupta et al. 2016]. Sua principal vantagem, assim como propõe o nome, é o uso reduzido de bateria, tornando possível que um dispositivo que utilize o padrão possa ficar ligado por anos com uma única carga de bateria.

O BLE pode ser utilizado como mecanismo de localização usando Received Signal Strength Indicator (RSSI) como forma de estimativa de distância e posicionamento. O mecanismo de localização utilizado neste artigo são os beacons, que também fazem uso do BLE.

\subsection{Bluetooth Low Energy Beacons}

Baseado no BLE, os bluetooth low energy beacons [Tanenbaum 2011] são pequenos dispositivos de baixa potência e, quando usam bateria, essas costumam durar por muito tempo, na faixa de anos. Em certas circunstâncias, estes dispositivos podem fornecer uma localização mais precisa do que o Global Positioning System (GPS), triangulação de torre de celular e, até certo ponto, que o Wi-Fi [Dasgupta et al. 2016]. Ao longo deste artigo, BLE beacons serão referenciados apenas como beacons.

Eles tem a capacidade de transmitir pequenas quantidades de dados via BLE. Se configurado no formato aberto Eddystone-URL [Mansell and Curran 2016], por exemplo, o beacon passa a transmitir uma URL para dispositivos próximos. Dentre os modelos de propagação existentes, destacam-se o Log-Distance Path Loss Model (LDPLM) e Rappaport's Path Loss Model (RPLM) [Galván-Tejada et al. 2013], [Rejane et al. 2011].

\section{Aplicativo PWA do Museu da Fauna e da Flora}

Com o intuito de explorar o conteúdo do MFF-ICMC, foi desenvolvida a aplicação Museu Digital da Fauna e Flora, um PWA. Ao estar próximo de um elemento do museu

\footnotetext{
${ }^{6}$ Bluetooth low energy https://www.bluetooth.com/what-is-bluetooth-technology/ how-it-works/low-energy
} 
VI Congresso Brasileiro de Informática na Educação (CBIE 2017)

Anais dos Workshops do VI Congresso Brasileiro de Informática na Educação (WCBIE 2017)

(que possui um beacon para sua identificação), o beacon enviará um link para a página específica deste elemento que encontra-se catalogado no Museu da Fauna e Flora, que, uma vez carregada a página, pode ser visto mesmo quando offline.

A interface do Museu foi implantada no Heroku (https:// digital-museum. herokuapp.com/) e seu código fonte pode ser encontrado no GitHub (https://github.com/hick209/digital-museum).

Para a realização do projeto ser mais fluida, ele foi dividido em algumas etapas. Essas etapas são apresentadas em detalhes nas subseções seguintes.

\subsection{Coleta de dados}

Uma etapa preliminar foi tirar fotos das placas de algumas das árvores encontradas no ICMC que compõem o MFF-ICMC (ilustrado na Figura 1). Fez-se isso a fim de se obter informações suficientes para iniciar a base de dados da aplicação, uma vez que ficou estabelecido que a base de dados de catalogação seria composta inicialmente pela flora, pela maior facilidade de aquisição e mesmo mapeamento. A base completa será posteriormente migrada para ser utilizada pelo aplicativo.

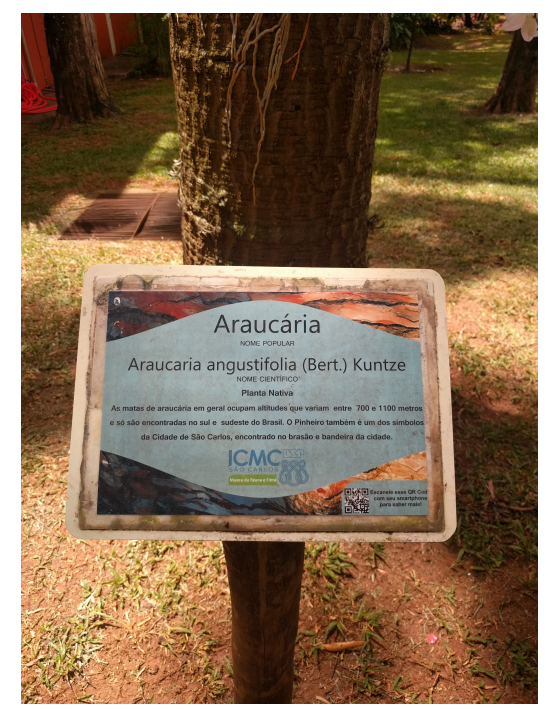

Figura 1. Exemplo de foto da placa de identificação de uma árvore do museu

\subsection{Construindo a aplicação}

A princípio, foi elaborado um estudo de qual a melhor abordagem: uma aplicação nativa ou uma aplicação na web. Optou-se por utilizar uma aplicação web, principalmente por proporcionar uma mobilidade e uma flexibilidade maior para o museu. Dentre os motivos que influenciaram esta decisão tem-se: estudo e uso de uma nova tecnologia para o desenvolvimento de museus virtuais (PWA); PWA é uma tecnologia de vanguarda, que faz uso do que há de mais moderno dentre as tecnologias web; Processo de aquisição de usuários mais curto, por não ser necessário instalar uma aplicação; Funciona em Android e iOS "out of the box" (sem esforço extra), além de outros sistemas.

Uma vez estabelecida a tecnologia de desenvolvimento, PWA, foi dado o início aos estudos das ferramentas e tecnologias necessárias para o desenvolvimento do projeto. 
Foi decidido usado o framework React + Redux, pois a curva de aprendizado e complexidade eram menores. Como ferramenta de build optou-se por utilizar Webpack pela simplicidade, além de permitir tratar todas as necessidades do projeto, como transpilar as versões mais recentes do ECMAScript e JSX para uma versão do JavaScript que todos os navegadores compreendem e também gerar um service worker a partir do projeto sem esforço adicional, o que é indispensável para o funcionamento da aplicação off-line.

Em relação à hospedagem da aplicação, utilizou-se o Heroku para fazer a implantação da aplicação. Como repositório de código, utilizou-se o GitHub, que depois foi conectado ao Heroku, para que ele pudesse fazer os deploys (implantações) diretamente a partir do repositório. A base de dados utilizada foi Firebase, por fornecer também outros serviços que são importantes para a aplicação, como autenticação e armazenamento de arquivos.

\subsection{Contextualizando a localização do usuário}

Em paralelo ao desenvolvimento da aplicação foi realizada uma análise de como a localização do usuário poderia ser utilizada para levá-lo até a página da aplicação referente ao elemento do museu. As opções analisadas foram: GPS dentro da aplicação; Beacons; Geofencing, porém a escolhida foi a tecnologia de Beacons, justificada pela facilidade de uso e boa integração com PWAs. Uma outra vantagem de utilizar beacons é que o usuário não precisa ter utilizado o aplicativo e concedido permissões para o mesmo, como seria necessário para utilizar Geofencing. Para isso basta estar próximo de um beacon.

\subsection{Usando os beacons}

Antes de utilizar um beacon faz-se necessária sua configuração, ajustando seu formato de comunicação, intervalo de broadcast de sinal e potência. A configuração escolhida foi: Formato Eddystone-URL, um intervalo de broadcast balanceado $(3 \mathrm{~Hz})$ e; potência baixa para prolongar a bateria e também para um alcance mais curto, dado que a intenção é apenas notificar quem estiver próximo do elemento.

O processo de configuração depende estritamente do fornecedor do beacon ${ }^{7}$, não existindo uma maneira única de configurá-los. Basicamente, cada fabricante possui um software para isso.

Se a URL não estiver aparecendo nos dispositivos receptores (normalmente celulares) que estão com o recurso de web física ligado, mesmo após ter sido feita a configuração do beacon, talvez seja necessário registrá-lo no Google. Isto é possível de ser feito por meio do aplicativo do Google, Beacon Tools ${ }^{8}$.

\subsubsection{Simulando um beacon}

Para testar ainda mais facilmente, é possível fazer uso de aplicativos simuladores de beacons, como ilustrado na Figura 2. Esses aplicativos são facilmente encontrados nas lojas

\footnotetext{
${ }^{7}$ Beacon configurations https://developers.google.com/beacons/get-started $\backslash$ \#2-configure-your-beacons

${ }^{8}$ Aplicativo Beacon Tools https://play.google.com/store/apps/details?id=com. google.android.apps. location. beacon. beacontools
} 
VI Congresso Brasileiro de Informática na Educação (CBIE 2017)

Anais dos Workshops do VI Congresso Brasileiro de Informática na Educação (WCBIE 2017)

de aplicativos. Neste projeto, foi utilizado o Beacon Simulator ${ }^{9}$.

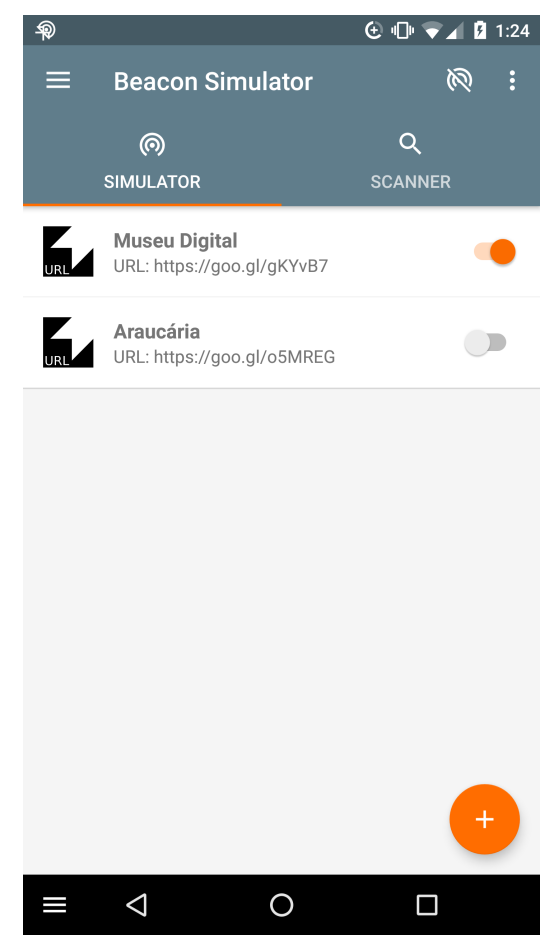

Figura 2. Tela inicial do Beacon Simulator

Após configurado o simulador, é possível visualizar a URL transmitida em outros dispositivos, como ilustrado na Figura 3.

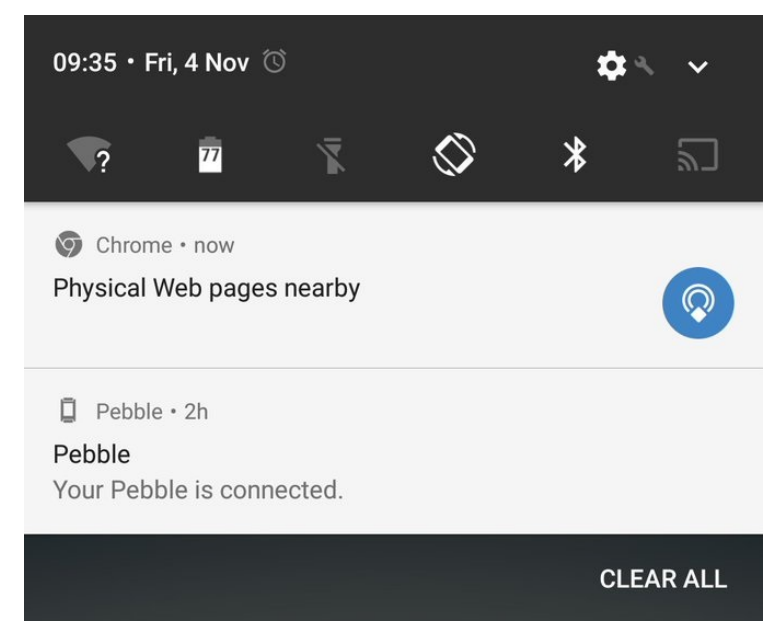

Figura 3. Notificação da Web física em um Android 7.0

\subsection{Configurando a web física}

Para visualizar as URLs emitidas por beacons sem necessitar, ativamente, abrir uma aplicação que procura por elas, é necessário ativar a Web física no dispositivo. Uma

\footnotetext{
${ }^{9}$ Aplicativo Beacon Simulator https://play.google.com/store/apps/details?id= net. alea.beaconsimulator
} 
VI Congresso Brasileiro de Informática na Educação (CBIE 2017)

Anais dos Workshops do VI Congresso Brasileiro de Informática na Educação (WCBIE 2017)

vez ativada, basta estar fisicamente próximo de um dos beacons (que podem ser itens do museu), reais ou simulados, que aparecerá uma notificação com o link emitido pelo beacon, assim como ilustrado na Figura 4.
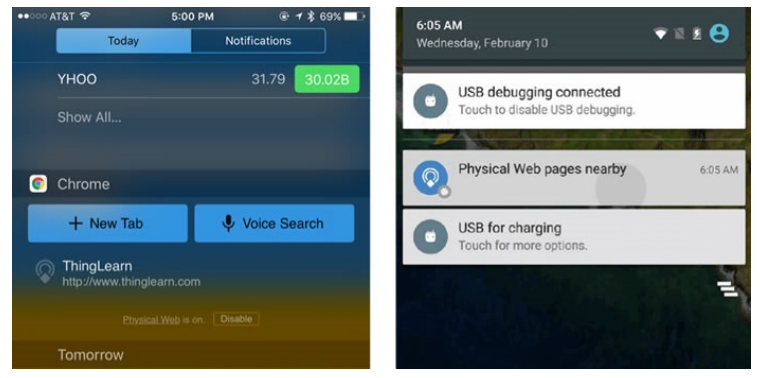

Figura 4. Exemplo de notificações da Web física

A web física, pode ser desativada a qualquer momento, caso o usuário deseje. Suas notificações ocorrem de forma silenciosa, isto é, não são acompanhadas de uma notificação sonora e nem mesmo, nos Androids, de um ícone na barra de status. Para visualizar a notificação, é necessário abrir a central de notificações.

É comum os Androids já virem com a Web física ligada, contudo como ele é um sistema aberto, é possível que o fabricante mude isso.

\subsection{Testando a aplicação}

Uma vez configurada a Web física em seu dispositivo, será possível utilizá-la como ponto de entrada da aplicação desenvolvida neste projeto, basta estar próximo de um beacon, real ou simulado, que emite a URL da aplicação, que encontrá-se disponível em https: //digital-museum.herokuapp.com/.

É possível acessar a aplicação diretamente, sem necessitar utilizar um beacon ou mesmo a web física, basta acessar sua URL. Acessando assim, será possível navegar no conteúdo existente da aplicação, como ilustrado na Figura 5.

\section{Resultados Obtidos}

Receber e acessar informações de acordo com o contexto atual em que se está inserido é útil, principalmente em aplicações sensíveis ao contexto. A metodologia utilizada neste trabalho é apenas uma das maneiras de explorar este conceito.

Foi escolhido desenvolver uma aplicação web, uma vez que o usuário não precisa instalar nada novo em seu dispositivo para interagir, neste caso, com o museu.

Uma vez que o objetivo do projeto é a criação de uma forma de interagir virtualmente com elementos do Museu da Fauna e Flora do ICMC-USP de São Carlos, pode-se dizer que ele foi alcançado, pois a plataforma para isso está disponível e com acesso irrestrito. Esta nova plataforma permite que um usuário, com um dispositivo com a Web física ligada, o que é padrão em muitos dispositivos, descubra o museu apenas por passar próximo de um dos elementos dele.

Não limitando-se a isso, o projeto também permitiu explorar conceitos como a Web física, beacons e PWA, que são assuntos que vêm ganhando espaço no mercado, especialmente PWA [Fransson and Driaguine 2017]. 
VI Congresso Brasileiro de Informática na Educação (CBIE 2017)

Anais dos Workshops do VI Congresso Brasileiro de Informática na Educação (WCBIE 2017)

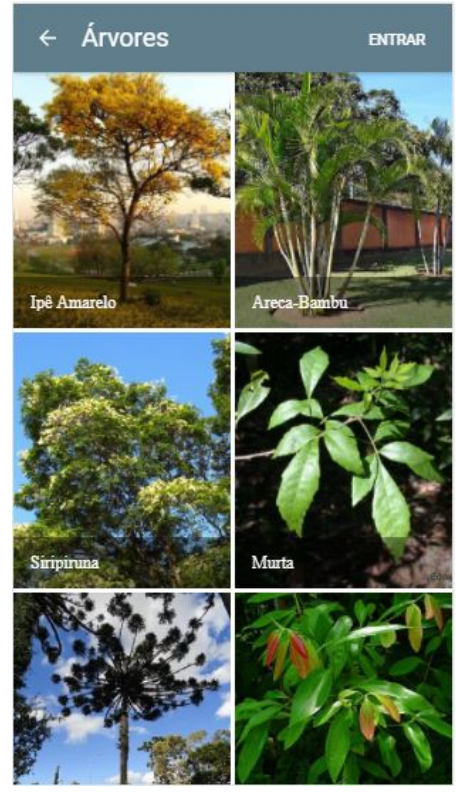

Figura 5. Visualização da lista de árvores do museu dentro da aplicação

\section{Conclusões}

Este trabalho criou uma plataforma que permite maior interação com o MFF-ICMC, apresentando informações sobre os elementos do museu. O desenvolvimento desta aplicação permite vislumbrar o uso de paradigmas de Internet das Coisas na educação, fazendo com que ocorra uma aproximação da realidade escolar e o emprego de tecnologias de informação e comunicação, possibilitando que ecomuseus posssam ser então utilizados na educação básica e fundamental.

Como trabalhos futuros pretende-se:

- Criar uma trilha a ser seguida pelos usuários da aplicação, explorando o acervo do MFF-ICMC.

- Adicionar crowdsourcing, com a finalidade de melhorar as informações do museu, permitindo inclusive que itens sejam incluídos pelos usuários.

- Incorporar essas tecnologias e funcionalidades em um processo que permite a geração de diferente interfaces dirigidas a contexto.

\section{Referências}

Atzori, L., Iera, A., and Morabito, G. (2010). The internet of things: A survey. Computer networks, 54(15):2787-2805.

Bell, K. M., Bleau, D. N., and Davey, J. T. (2011). Push notification service. US Patent $8,064,896$.

Brulon, B. (2015). A invenção do ecomuseu: O caso do écomusée du creusot montceaules-mines e a prática da museologia experimental. Mana, 21(2):267-295.

Dasgupta, A., Nagaraj, R., and Nagamani, K. (2016). An internet of things platform with google eddystone beacons. Journal of Software Engineering and Applications, pages 291-295. 
VI Congresso Brasileiro de Informática na Educação (CBIE 2017)

Anais dos Workshops do VI Congresso Brasileiro de Informática na Educação (WCBIE 2017)

Fransson, R. and Driaguine, A. (2017). Comparing progressive web applications with native android applications: An evaluation of performance when it comes to response time.

Galván-Tejada, C. E., Carrasco-Jiménez, J. C., and Brena, R. F. (2013). Bluetooth-wifi based combined positioning algorithm, implementation and experimental evaluation. Procedia Technology, 7:37-45.

Gaunt, M. (2017). Service worker: an introduction.

Mansell, G. and Curran, K. (2016). Location aware tracking with beacons.

Rejane, D., Thierry, V., and Adrien Van Den, B. (2011). Comparison of indoor localization systems based on wireless communications. Wireless Engineering and Techno$\log y, 2011$.

Sparrow, B., Liu, J., and Webner, D. M. (2011). Google effects on memory: Cognitive consequences of having information at our fingertips. ScienceMag, 333(6043):776778.

Tanenbaum, A. (2011). Computer networks, /andrew s. tanenbaum, david j. wetherall. Cloth: Prentice Hall. 